\title{
Even flavor QED3 in an external magnetic field
}

\author{
K.Farakos and G.Koutsoumbas ${ }^{\mathrm{a}}$, N.Mavromatos and A.Momen ${ }^{\mathrm{b}}$ \\ a Physics Department, National Technical University, \\ Zografou Campus, 157 80, Athens, Greece \\ ${ }^{\mathrm{b}}$ Department of Physics, Theoretical Physics, \\ 1 Keble Road,Oxford OX1 3NP, U.K.
}

The magnetically induced fermionic condensate is studied at zero and finite temperatures. The effects of a non-homogeneous external magnetic fields is briefly considered.

\section{INTRODUCTION}

Considerations of the effects of external magnetic fields in the Early Universe and in problems of high $T_{c}$ superconductivity ([1], 2]) are the main motivations to study the behaviour of the fermionic matter under the influence of an external magnetic field.

The three-dimensional continuum Lagrangian of the model is given by:

$\mathcal{L}=-\frac{1}{4}\left(F_{\mu \nu}\right)^{2}+\bar{\Psi} D_{\mu} \gamma_{\mu} \Psi-m \bar{\Psi} \Psi$

where $D_{\mu}=\partial_{\mu}-i g a_{\mu}^{S}-i e A_{\mu} ; a_{\mu}^{S}$ is a fluctuating gauge field, while $A_{\mu}$ represents the external gauge field. The main object of interest here is the condensate $\langle\bar{\Psi} \Psi>$, which is the coincidence limit of the fermion propagator, $S_{F}(x, y)$.

\section{RESULTS IN THE CONTINUUM}

A first estimate for the enhancement of the condensate arising from the external fields may be gained through the analysis of the relevant Schwinger-Dyson equation:

$$
\begin{gathered}
S_{F}^{-1}(p)=\gamma \cdot p \\
-g \int \frac{d^{3} k}{(2 \pi)^{3}} \gamma^{\mu} S_{F}(k) \Gamma^{\nu}(k, p-k) D_{\mu \nu}(p-k)
\end{gathered}
$$

where $\Gamma^{\nu}$ is the fermion-photon vertex function and $D_{\mu \nu}$ is the exact photon propagator ( [5]).

The results of a recent approximate solution of the above equation in the regime of small homogeneous external magnetic field, both for quenched

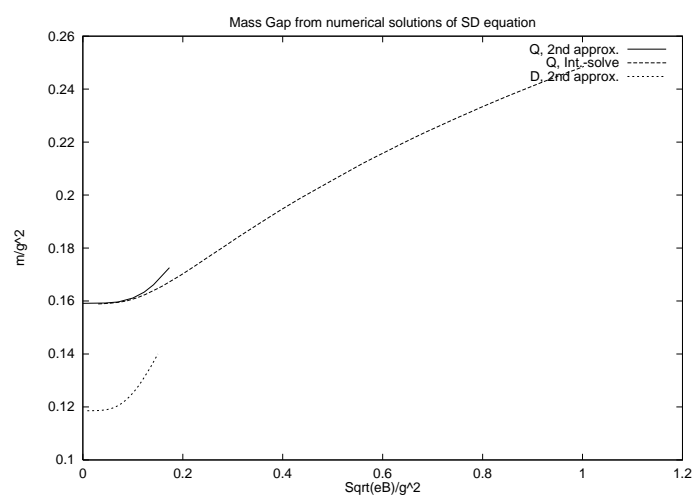

Figure 1. Solution of Schwinger-Dyson equation for quenched and dynamical fermions.

and dynamical fermions are depicted in figure 1, where one may see the dynamical mass generated, versus the magnetic field strength. The upper curve (labeled "Q,Int-solve") is the solution for quenched fermions, while the lower curve is the weak field approximation to the dynamical fermionic condensate. We have also included the weak field approximation to the quenched result, as a measure of the reliability of the weak field expansion.

There have also been approximations in the regime of strong magnetic fields [3], but for a fully quantitative treatment one should rely on the lattice approach $($, 1,5$])$. 


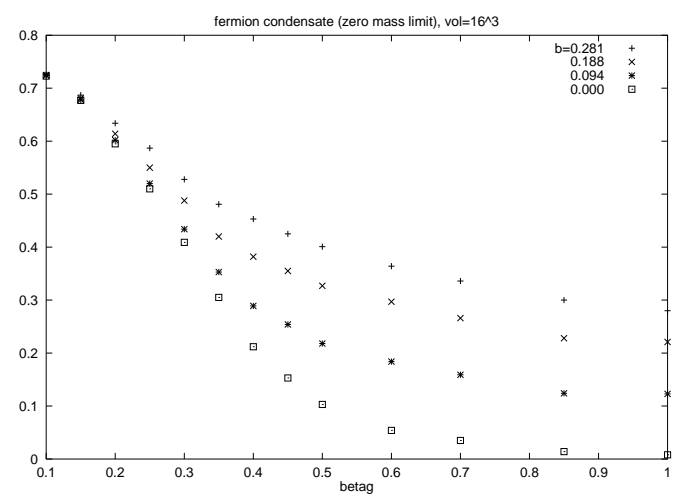

Figure 2. $<\bar{\Psi} \Psi>$ versus $\beta_{g}$ for various values of the magnetic field.

\section{LATTICE RESULTS}

We will first present the results for the $T=0$ case and a homogeneous magnetic field. Figure 2 contains the condensate versus the gauge field coupling constant, $\beta_{g}$, for several values of the magnetic field. This figure is the final outcome of a series of measurements performed at several values of the bare mass; the result shown here is the extrapolation to the zero mass limit. The result is independent from the magnetic field at strong gauge coupling, because the gauge interactions are the main contributor to the condensate in this regime; the magnetic field takes over in the weak coupling, on the right hand part of the diagram.

Figure 3 contains the condensate versus $\beta_{g}$ for a typical fixed value of the magnetic field. The uppermost data correspond to a symmetric lattice $16^{3}$. The curve is smooth and no sign of discontinuity can be seen anywhere. The next result comes from an asymmetric lattice, with a rather large time extent, though: $24^{2} \times 6$. A structure starts showing up at $\beta_{g} \approx 0.45$. To see better this structure, we go to the $16^{2} \times 4$ lattice; the structure moves to $\beta_{g} \approx 0.40$ and becomes somehow more steep. The effect of the spatial size of the lattice is not big, as one may see by comparing the points for $16^{2} \times 4$ versus the ones for $24^{2} \times 4$,

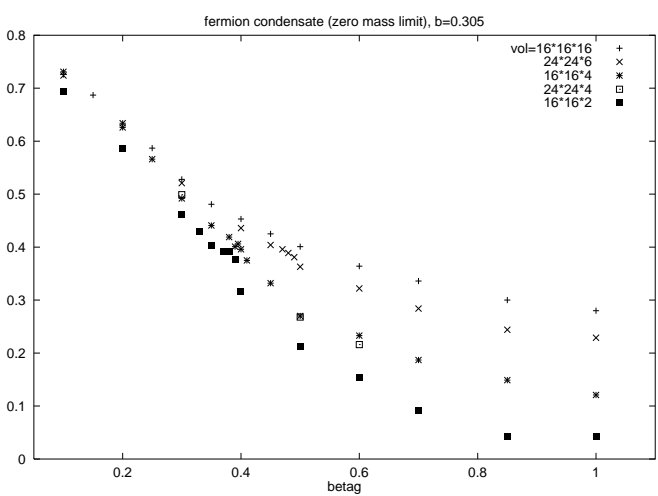

Figure 3. $<\bar{\Psi} \Psi>$ versus $\beta_{G}$ in the zero mass limit at various tamperatures $\frac{1}{N_{t}}$.

which are also shown on the figure. Finally the points for $16^{2} \times 2$ show a clear discontinuity in the slope. Although it deserves more detailed study, it seems safe to interpret this discontinuity as a symmetry restoring phase transition, imposed by the nonzero temperature.

In figure 4 we use the results for various lattice sizes to construct a graph showing the dependence of the condensate on the temperature for two values of the external magnetic field. It is surprisingly similar to the corresponding result for the "free" case (沺). The condensate tends to zero for large temperatures; this tendency is more intense for the smallest magnetic field. In the last figure we show the results of a study of a non-homogeneous magnetic field. We consider a lattice in which its central $6 \times 6$ region (for all values of z) carries a constant magnetic flux, while in the rest part the magnetic field vanishes ( $[5])$; we measure the condensate along a straight line passing from the center of the lattice at a fixed value of the magnetic field. This profile is shown in figure 5 and one may see that the condensate is non-zero only in the region of non-vanishing magnetic flux. Away from this region the remaining condensate may be accounted for by the explicit mass term and has little to do with the external magnetic field. 


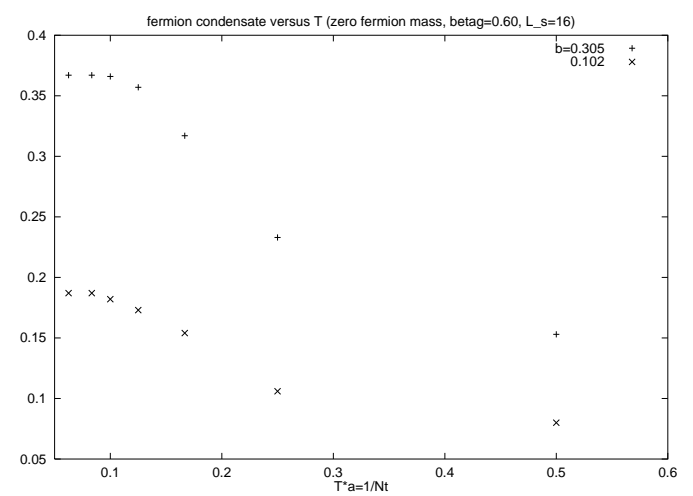

Figure $4 .<\bar{\Psi} \Psi>$ versus the temperature for two values of the magnetic field.

\section{Acknowledgements}

K.F. and G.K. would like to acknowledge financial support from the TMR project "Finite temperature phase transitions in particle Physics", EU contract number: FMRX-CT970122. The work of N.E.M. is partially supported by PPARC (UK) through an Advanced Fellowship. The work of A.M. is supported by PPARC.

\section{REFERENCES}

1. N.Dorey and N.E.Mavromatos, Nucl.Phys. B386 (1992) 614.

2. K.Farakos and N.E.Mavromatos, Int.J.Mod. Phys. B12 (1998) 809.

3. A.V.Shpagin, Dynamical mass generation in $(2+1)$ dimensional electrodynamics in an external magnetic field, hep-ph/9611412.

4. K.Farakos, G.Koutsoumbas and N.E.Mavromatos, Phys.Lett. B431(1998)147.

5. K.Farakos, G.Koutsoumbas N.E.Mavromatos and A.Momen, On magnetic catalysis in even-flavour QED3, hep-ph/9905272.

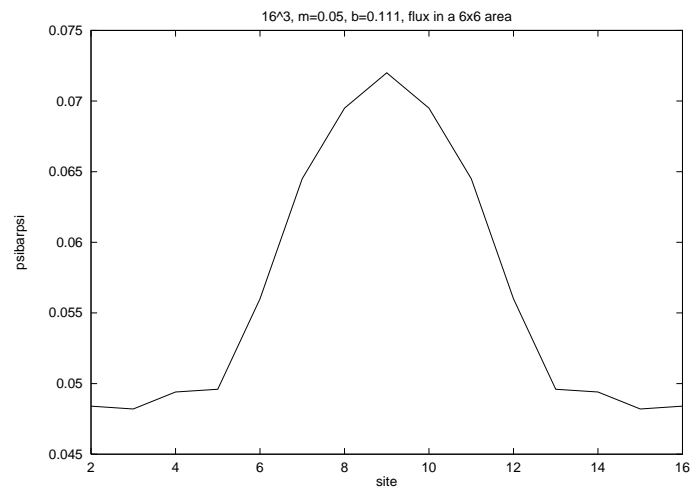

Figure 5. $<\bar{\Psi} \Psi>$ along a straight line passing from the center of the lattice if the magnetic field parameter $b$ is set to 0.111 . The central region of non-zero flux is $6 \times 6$. 